\title{
Infusing the axioms of clinical reasoning while designing clinical anatomy case vignettes teaching for novice medical students: a randomised cross over study
}

\author{
Dinesh Kumar. $\mathrm{V}^{1}$, Rajprasath $\mathrm{R}^{2}$, N.A. Priyadharshini ${ }^{3}$, Magi Murugan $^{2}$, Rema Devi $^{2}$ \\ ${ }^{1}$ Department of Anatomy, Jawaharlal Institute of Postgraduate Medical Education and Research, Puducherry, ${ }^{2}$ Department of Anatomy, Pondicherry \\ Institute of Medical Sciences, Puducherry, ${ }^{3}$ Department of Anatomy, Sri Manakula Vinayagar Medical College and Hospital, Puducherry, India
}

\begin{abstract}
The clinical reasoning skills is often gained when the biomedical knowledge is broadened and deepened alongside exposure to patients. The 'ideal' blend of axioms of clinical reasoning and case based learning would establish the pedagogical bridges right from the first year of medical education. So this study aimed to investigate the perceived importance and efficacy of teaching clinical reasoning skills among first year medical students, as this has not previously been described. As a priori, two clinical reasoning skill sessions were conducted using clinico-anatomical case vignettes designed according to the literature regarding clinical reasoning ('serial cue' approach and hypothetico-deduction). Students were divided into intervention and control group and crossed over in subsequent sessions. Analysis was done by mixed method approach including measuring proof of benefit using post-test comparison, quantitative survey and qualitative analysis by nominal group discussion. Post test scores were compared using student's $t$-test. Feedbacks were analysed using descriptive statistics. The results showed that post test scores were significantly higher in intervention group than the control group in both sessions $(P<0.001,0.016)$. A total of $66 \%$ students felt, diagnostic skills and lateral thinking abilities were improved and It helped in developing problem-solving abilities for $67 \%$ students. clinico-anatomical case vignettes helped in understanding anatomical basis of clinical conditions for $61 \%$ students. To conclude, introducing clinical reasoning has considerable effect in improving the decision making ability of the students and if incorporated right from the first year, would better prepare the students in successful transition to clinical learning environment.
\end{abstract}

Key words: Clinical reasoning, Problem-based learning, Case vignette, Novice learner, Mixed methods

Received September 18, 2019; Revised December 10, 2019; Accepted December 19, 2019

\section{Introduction}

The ability to make accurate clinical diagnosis is one

Corresponding author:

Rajprasath R (iD)

Department of Anatomy, Pondicherry Institute of Medical Sciences, Puducherry 605014, India

E-mail: rajprasathanat@gmail.com of the critical elements of patient care. The preciseness of reasoning hugely depends upon translating the biomedical knowledge gained in the pre-clinical phase of medical education into clinical acumen. The contemporary medical education fraternity places much emphasis on teaching of clinical reasoning skills right from the first year of medical education [1]. However, due to variations in the curriculum across the globe, there is a lack of concrete evidence on which methodology works better and when it should be implemented [2] We could perceive the changes in the pre-clinical medical

\section{Copyright (C) 2020. Anatomy \& Cell Biology}

This is an Open Access article distributed under the terms of the Creative Commons Attribution Non-Commercial License (http://creativecommons.org/licenses/by-nc/4.0/) which permits unrestricted non-commercial use, distribution, and reproduction in any medium, provided the original work is properly cited. 
education over the past few years in terms of the shift from traditional discipline specific approach to an integrated case based approach. The traditional case based approach principally eyes on increasing the relevance of the learned content in clinical scenarios and harnessing the problem solving skills. It operates based on the principle that effective learning takes places when individuals are made to apply knowledge and skills to real life scenarios $[3,4]$. Literature $[1,5,6]$ suggest that, increasing the authenticity of the cases would expose the students to ambiguity and complexity of the realworld clinical settings.

The Liaison Committee on Medical Education defines clinical reasoning as "the integration, organization, and interpretation of information gathered as a part of medical problem-solving" [7]. These skills are emphasized because a graduate medical student should be able to prioritize the differential diagnosis following a clinical encounter and draw conclusions [8]. In other words, it is an inferential process involving collection and analysing of data for making judgements/decisions regarding the diagnosis of clinical problems. However, evidence suggests that the progressive development of clinical reasoning skills in medical students is often inadequate and this can be largely attributed to the unstructured education imparted in this domain [9].

The problem presentation in traditional case based learning suffers from the process of distilling critical information whereby broader clinical scenario are simplified using lot of semantic qualifiers [10]. When problems are explicitly represented, the session leaves less scope for reasoning abilities. This could be stated as one of the major discrepancies between cognitive skills which are required for application in realistic settings and those problems illustrated through conventional case based sessions [11, 12]. Further, the factor of hindsight bias contaminates the objectives of these sessions [13]. Hindsight bias props up when students, who know the correct diagnosis from the initial clues of the case, tend to overestimate the likelihood of diagnosis when they are placed in a prospective setting. When students are provided with full content of the case, they don't undergo the iterative process of reasoning and unless they are exposed to uncertainties, they wouldn't be able to compare the diagnostic probabilities in the setting of conflicting or ambiguous clinical data $[2,12,14,15]$. In spite of its significance, we can enunciate that pre-clinical years seldom provide sufficient opportunities for developing clinical reasoning [16].

Students, in the pre-clinical phase of medical education gain more theoretical knowledge related to anatomical structures (anatomy) and their functions (physiology). When they are asked to deliberately reflect upon the paper based cases, as in traditional case based learning, they try to analytically reason it out. However, for understanding of the cause-effect relationships between signs and symptoms of a disease, the core anatomical knowledge needs to be encapsulated [17]. Thus, it is pertinent to develop broader causal models which are similar to illness script concordance tests. While acquainting the scripts, even novice students tries to integrate biomedical knowledge and clinical pictures [18]. The intended positive effect of exposing novice students to 'embryonic' model of scripts is that, they get stored in students' memory in the form of mental representation of a disease along with required biomedical knowledge and clinical presentation [19]. This mental representation get activated by patients' cues, the diagnostic reasoning process gets stimulated and would guide them. If we extrapolate this, we could posit that diagnostic performance of the clinician depends upon the amount and richness of the illness scripts available in his/her memory [19].

Although several strategies have been described in the literature, there is no single 'gold standard' for teaching clinical reasoning for first year medical students. In the light of abovementioned necessitate for stand-alone clinical reasoning session in the pre-clinical year, we have developed clinico-anatomical vignette based sessions for analysing the clinical cases. The vignettes, we had used, had the benefits of both problem representation and illness scripts. For solving the case, students need to compare alternative diagnoses and garner the evidence from the case which would fall in line with the diagnosis [20]. The continuum of clinical reasoning experience, over here, is based on two factors: a) how student reaches the diagnosis via appropriate reasoning b) how he/ she uses the knowledge underlying the above said reasoning process by choosing the relevant details and organizing it into a coherent form [21].

We hypothesized that this newer approach would increase the interest of students and hone their clinical reasoning ability, both analytical and cue based. This pilot study was implemented at our institute as a small group exercise during the first year. The objectives are to evaluate the set of outcomes during clinical reasoning training. Specifically, we aimed to document the interest, engagement and perceived gain of knowledge between novice students who have been exposed to vignettes and those who haven't. 


\section{Materials and Methods}

\section{Setting and participants}

The study design was approved by the institute research and ethical committee (ethics approval number: IEC: $\mathrm{RC} / 17 / 58)$. All 150 first year medical students at Pondicherry Institute of Medical Sciences were invited to participate in the study on voluntary basis. Students underwent clinical reasoning training for the first time and the sessions were conducted after they have been exposed to traditional anatomy classes of that particular region by means of lectures and dissection classes. The students were briefed about the study design, objectives and recruited as participants after obtaining written consent. The pilot study was run as an extra-class activity in two sessions (one hour each) offered on different days to fit in ongoing timetable.

\section{Design}

The pilot study involved random allocation of participants to either study group or control group. The study group was subjected to clinical reasoning session which consisted of following interactive segments: 1) outline of the segments 2) nutshell of the clinical case, 3) gradual unwinding of the case from the time of admission to post-operative period 4) probe questions in each of the segments 5) organization of the case [22]. At the end of the session, the study group was evaluated using post-test, which principally consisted of reasoning type of questions from the particular topic. The control group was first subjected to the test and later subjected to clinical reasoning session. In the second session, which was conducted in a different day, the groups were cross-overed so that the study group of the previous session acted as control group and vice-versa.

\section{Reasoning principles of vignettes}

For each vignette, students were asked to follow sequential steps. This involved 'serial-cue' approach, a way of hypothetico-deductive reasoning similar to real-life clinical settings [23]. When the chief complaint is presented, students were asked to generate diagnostic hypotheses and deduce potential consequences they would expect in course of the case (Fig. 1). The additional information was disclosed in sequential basis in the due course and students were asked to deliberately reflect upon them.

The history, consisting of relevant details along with few trivial information, was first displayed and students were

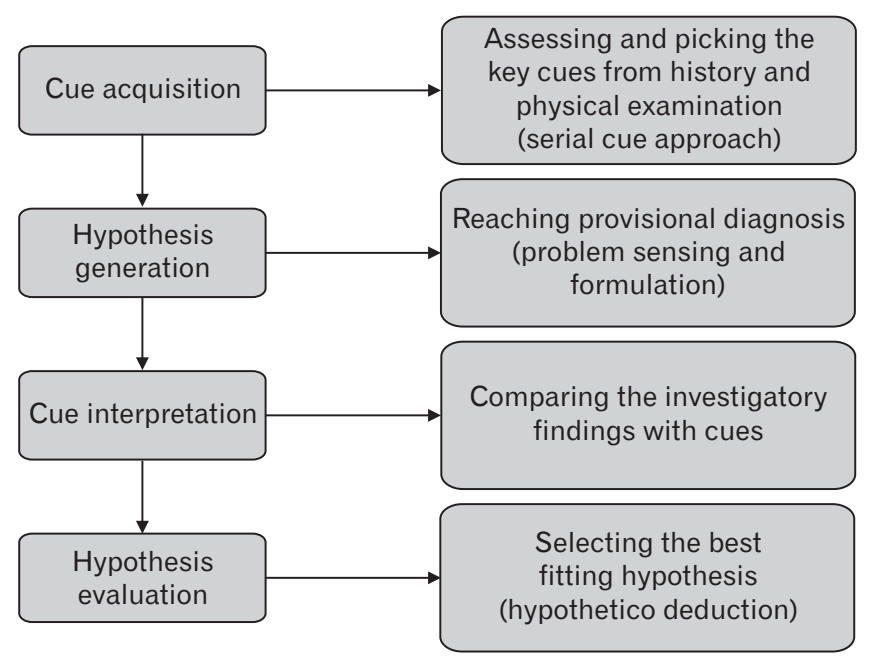

Fig. 1. Concept map of clinical reasoning principles used in the vignettes.

asked to write down one or more diagnoses that would come to their mind upon reading it. Two probe questions, based on reasoning ability related to findings in the history, were displayed and students were asked to discuss in groups of three ('think-pair-share' approach) and the resultant interpretation was collated. Following this, physical examination findings were displayed and students were asked to either substantiate or rule out the already generated hypotheses. This was followed by displaying of laboratory and radiological tests. Based upon this, students were asked to arrive at the final diagnosis (Fig. 2). Probe questions were displayed at each segment and collaborative discussion was promoted. Apart from discussion questions, remaining steps demanded selfexplanation only. Students were allowed to spend as much time as they needed.

\section{Preparation of vignettes}

Two cases (varicose veins and thyroid goitre) were chosen based on the prevalence of disease in our settings. Further, the cases were chosen on the basis that a novice student could interpret the provided history, physical examination findings and laboratory investigation and would also discuss the basic management steps based on anatomical knowledge itself. The information was compiled into a Microsoft PowerPoint (Microsoft, Redmond, WA, USA) presentation by one investigator and edited by another investigator. The content was vetted for its chronological sequence similar to the pattern encountered by the treating health care professional. 


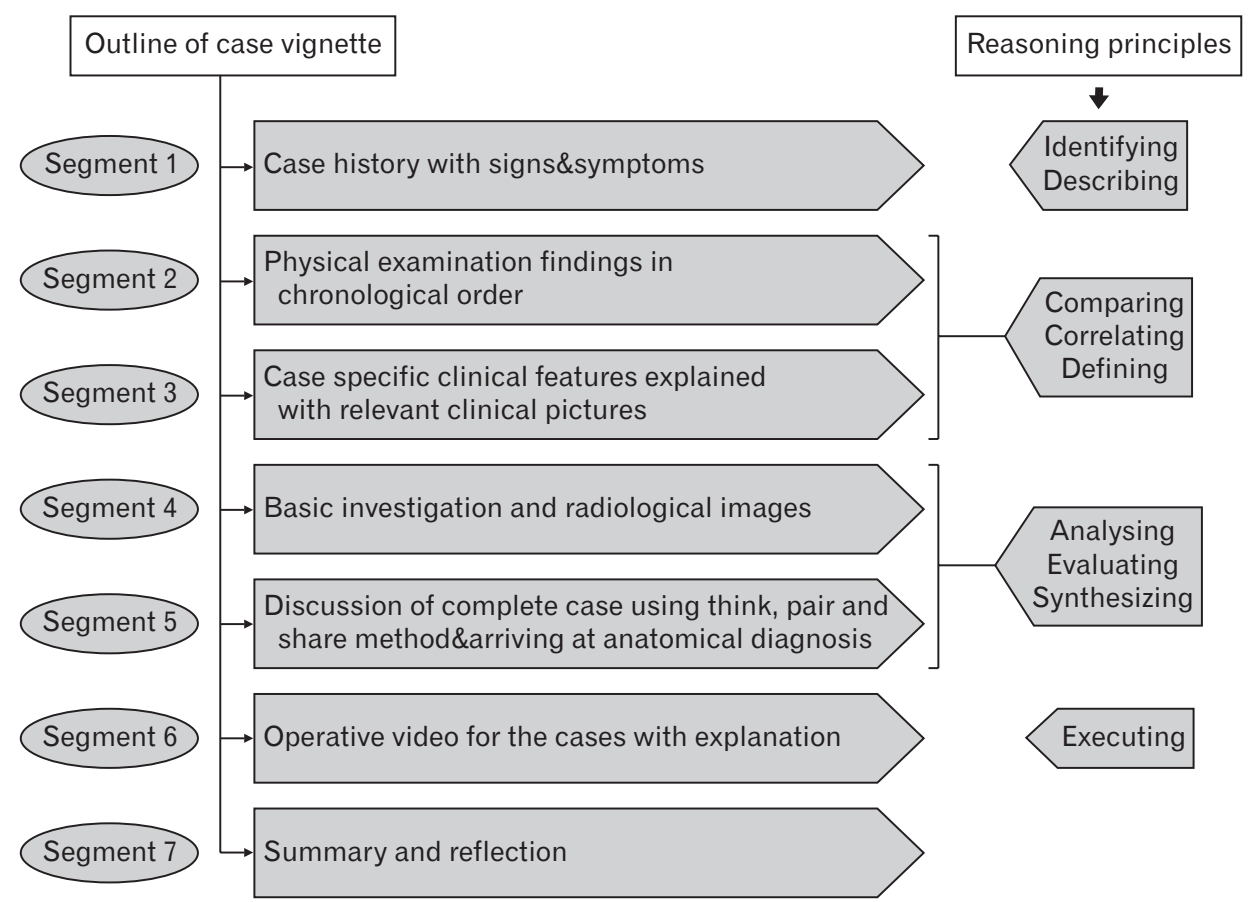

Fig. 2. Outline of segments and the reasoning principles used in the process of clinical anatomy case vignettes.

\section{Conducting the sessions}

Each session was facilitated by the principal investigator who had presented the case. The students and two of the investigators, who were in charge of conducting the evaluation, were blinded to the case content before the commencement of the session, including the diagnosis. The facilitator prompted the students to interpret each set of information provided in individual segments while moving sequentially through the vignette. Students were also encouraged to ask questions in the process.

\section{Overview of the modules used in the study}

\section{Varicose vein case module}

\section{Step 1: History}

- A 50 years old male came to outpatient department (OPD) with complaints of dull aching and heaviness over the left leg region. His symptoms are increasing throughout the day and relieved by taking rest with elevated legs

PQ1: What do you think he is suffering from?

PQ2: What is the next history you want to ask?

- His occupation is bus conductor; He is also complaining of swelling of ankle during prolonged standing and worm like long swellings in the leg region
PQ1: What is your provisional diagnosis and why do you think so?

PQ2: What is the cause of ankle swelling?

Step 2: Examination: Inspection

- Picture showing engorged dilated veins over the medial aspect of leg and lower medial thigh was displayed

PQ1: What is your inference?

$\mathrm{PQ2}$ : What is the anatomical reason for this finding?

- Picture showing the skin over the lower part of leg was thick \& darkly pigmented

PQ: What is the reason behind pigmentation of skin in this case?

- Surgeon inspected anterior abdominal wall and no abnormality seen in the anterior abdominal wall

PQ: What is the link between anterior abdominal wall and this case?

Step 3: Palpation

- Surgeon checked the dorsalis pedis artery pulsation over dorsum of foot and found to be normal (with picture)

PQ: Why did the doctor did so? What is its importance in this case?

- Surgeon did per-abdominal and per-rectal examination and no abnormality was found 
PQ: What is the clinico-anatomical reason for doing this examination in this case?

- The surgeon asked the patient to lie down and the veins are emptied. Then he compressed his thumb $3.5 \mathrm{~cm}$ below and lateral to pubic tubercle and asked the patient to stand up. He waited for 1 minute and the veins were gradually filling up from below

PQ: What is the name of the test performed by the doctor? Why this test is being done? What is your inference from this test?

- The surgeon asked the patient to lie down and the veins are emptied. Then he tied a tourniquet around upper thigh and asked the patient to stand up, checked for venous filling. He repeated the test with placing the tourniquet in midthigh and over calf region.

PQ: What is the name of the test performed by the doctor? Why this test is being done?

- The surgeon asked the patient to lie down and the veins are emptied. Then he tied a tourniquet around upper thigh and asked the patient to stand up and do mild exercise, and the veins become distended and the distension increased with exercise

PQ: What is the name of the test performed by the doctor? Why this test is being done?

Step 4: Investigations

- Routine blood investigations were within normal limits (complete blood count, hemoglobin)/chest x-ray was normal

- Doppler ultrasonogram (USG) picture and videos of procedure and results were shown and discussed

Step 5: Management

- Medical and surgical managements for Varicose veins were discussed

- Surgical video of ligation and stripping procedure with labelling and probe questions and newer techniques of laser ablation/foam sclerotherapy were shown and discussed

Step 6: Post-operative care and discharge advice related to this case and anatomical reasons were discussed
Thyroid goiter case module

Step 1: History

- Mr VM, a 45 year old male visits the surgical OPD, complaining that he could notice a visible swelling in the neck. He said that the swelling had gradually increased in size over the period of 6 months. He also complained that his voice has become hoarse over the period of 3 months

PQ: What are the possible reasons for hoarseness of voice?

- On probing, he admits that he has difficulty in breathing at the times of intense work.

PQ: What is the possible reason for this?

Step 2: Examination: Inspection

- The surgeon observed a swelling of size $2 \times 3 \mathrm{~cm}$ in front of the neck.

PQ: Can you mention few structures which can produce a swelling in this area?

- The surgeon asks the patient to put his tongue out and to swallow. The swelling ascended up and moved with deglutition.

PQ: What is the reason behind this?

Step 3: Palpation

- On further examination, the upper border of the swelling could be observed closer to the laryngeal prominence and the lower border of the swelling cannot be palpated (with picture)

PQ: What might be the reason behind this?

- On asking the patient to elevate his arms, he had facial plethora and dilated veins over the neck (with picture)

PQ: Explain the anatomical basis for this feature

- The trachea appears to be shifted towards the right side and there was a variation in the localization of carotid pulse on comparing both sides.

PQ: Explain the anatomical basis for this clinical feature

Step 4: Investigations: After arriving at a provisional diagnosis, the surgeon ordered for a battery of laboratory tests to determine the functional activity.

- The chest $\mathrm{x}$-ray postero-anterior view of the patient was shown and asked to identify the abnormality

- USG picture was shown and asked to identify the structures and abnormality 
- Labelled USG video on thyroid gland was shown and discussed

- To determine the extent and functional activity of thyroid gland scintigraphy was done - picture shown and the inference was discussed

- Fine needle aspiration cytology image was shown and asked to identify the procedure

- Computed tomography images - axial section, sagittal sections were shown and asked to identify anatomical structures related to the case

\section{Step 5: Management}

- Surgical incisions needed was explained with picture

- Surgical procedure explained step by step with pictures

- Surgical video with labelled structures was displayed with probe questions to identify the structures

Step 6: Postoperative complications

- Twitching or spasms of your muscles, Paraesthesia and convulsions

PQ: What is the structure affected and why do you think so?

- Voice change after ex-tubation

$\mathrm{PQ}$ : Why there was voice change?

\section{Measuring the effectiveness of the clinical reasoning session}

The evaluation was done at two levels

- Students reaction to the session (acceptance/perceived usefulness) was elicited

A) By asking them to rate the usefulness using quantitative items.

Individual interest in clinical reasoning was measured and students could respond to these items on a fivepoint Likert scale ranging from 'strongly disagree' to 'strongly agree'. The items were verified for internal construct reliability (Cronbach's alpha - 0.84).

B) By snapshotting perception of students about the proceedings of clinical reasoning session using nominal group technique. For this, students were divided into subgroups consisting of 15 students with one faculty facilitator each. Students were asked to reflect individually on the perceived benefits of clinical reasoning sessions and on the ways in which the session helped in solving clinical problems. In the second step, the individual responses are collated. Subsequently, the collated responses are rationalized and list of responses were prepared in each subgroup. The top five responses from each of the subgroup were collected and tabulated. After omission of replicative responses, the statements were arranged according to the frequency of representation.

- The learning gains was measured by comparison of post-test scores (Kirkpatrick level 2: proof of benefit): The post-test question paper was prepared by one of the investigators who is blinded to the learning content. It had 12 questions [3- fill in the blank type (for factual knowledge); 3- clinical scenario based MCQs, 3 - fill in the blank type (for clinical reasoning) and 3- true or false (scenario/factual)] with a maximum mark of 24 .

\section{Statistical analysis}

The proof of benefit was measured by comparing the post-test scores of both groups for each session using Student's $t$-test (independent). A $P$-value of $<0.05$ was considered statistically significant in a 2 -tailed test. The responses for quantitative items were summated.

\section{Results}

Post test scores comparison between intervention group (75) who underwent clinical reasoning sessions and control

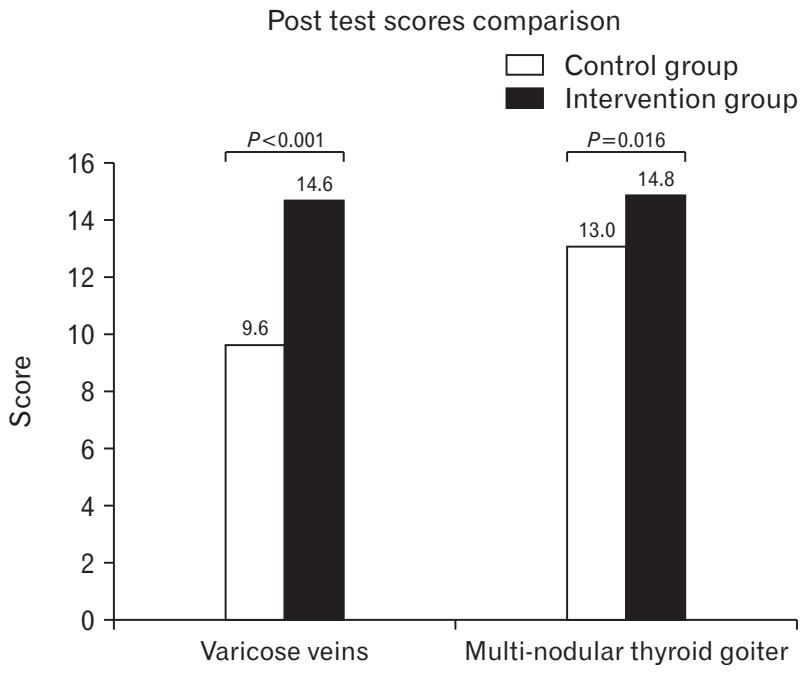

Fig. 3. Comparison of mean post test scores between intervention and control groups. 
group (75) who weren't exposed is shown in Fig. 3. Mean scores were significantly higher in intervention group compared to control group.

Mean perceptions of the students on the effectiveness of clinical reasoning sessions are summarized in Figs. 4, 5. When asked about the organization of contents of the sessions, $88 \%$ students agreed to the fact. A total of $71 \%$ students agreed that the probe questions asked during the sessions facilitated the discussion in small groups whereas $7 \%$ of students disagreed and $22 \%$ were neutral to it. A total of $25 \%$ students agreed and $72 \%$ strongly agreed that the clinical anatomy case vignettes were relevant to the context. Clinical reasoning sessions helped $66 \%$ students in proving diagnostic and lateral thinking skills and $83 \%$ students agreed that the discussion sessions have facilitated interaction with the peers as well as with faculties. A total of $61 \%$ students strongly agreed that the clinical anatomy sessions helped in better understanding of anatomical basis of clinical conditions.

\section{Student's perceptions from free text responses on individual basis are collated as follows}

Positives about the module

1. "Helped in applying theoretical knowledge in an applied way"

2. "Clinical videos which were displayed helped us to retain long term memory"

3. "Integrated clinical based learning helps us to correlate different pieces of knowledge"

4. "The interaction with peers helped us in effective team building"
5. "The clinical images and vignette showed the way in which diagnosis would be made in real life settings"

6. "Exposure to clinical based questions would help us in solving clinical scenarios in future"

\section{The common reflections of students on the reasons for incorporating the clinical reasoning sessions in the anatomy curriculum}

Do you feel that clinical reasoning sessions should be incorporated in anatomy curriculum? If so why?

1. It helped us in making better clinical correlation of the content learnt by conventional teaching

2. It kindled the curiosity in us

3. The interaction and reaching the diagnosis made us enthusiastic

Do you think that clinical reasoning sessions help in improving the problem solving abilities?

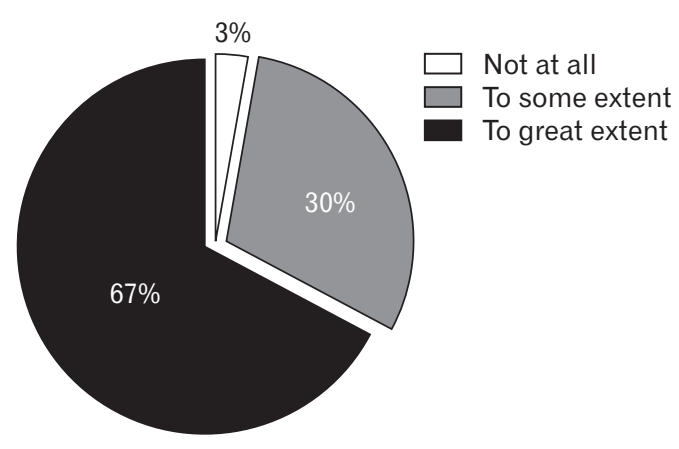

Fig. 5. Students reflection on usefulness of clinical reasoning sessions in improving problem solving abilities.

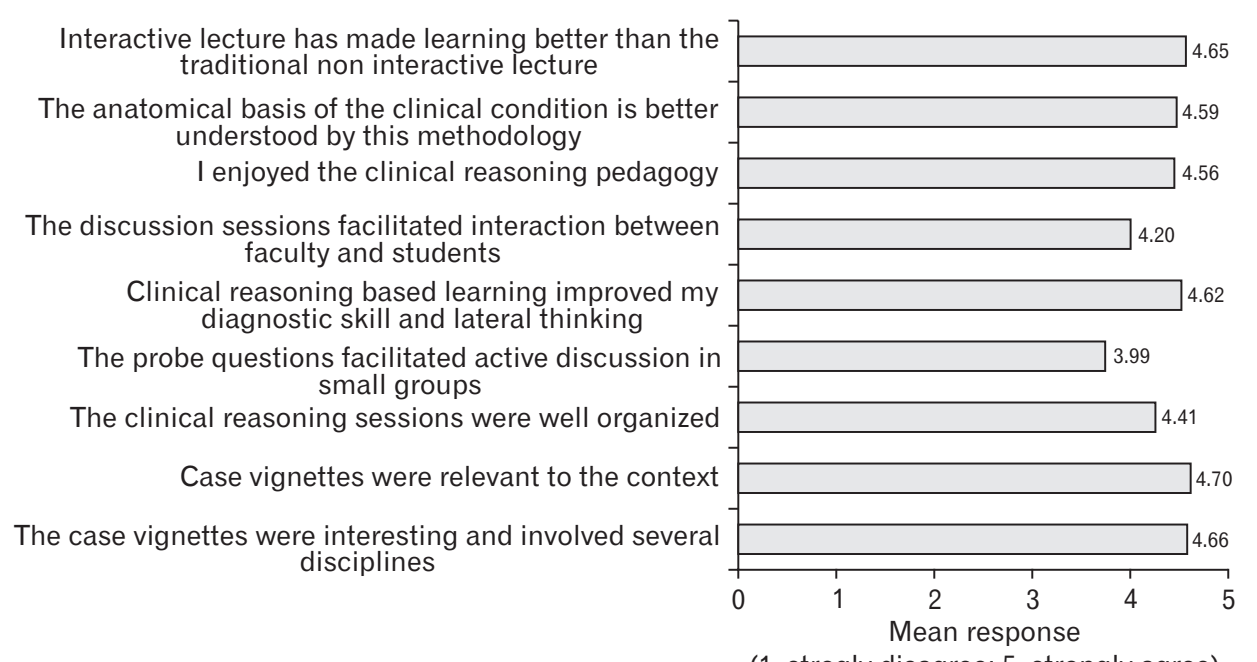

(1, strogly disagree; 5 , strongly agree)
Fig. 4. Perceptions of students on the effectiveness of clinical reasoning sessions. 
4. It helped in gaining better and wholesome orientation of the subject

5. It was useful in developing my diagnostic skills

6. I feel it makes the subject to be learnt in an easier way

7. The sessions made me generate different ideas

\section{Results}

Merits of clinical reasoning sessions (top five responses after collation and arranged according to frequency)

1. Better correlation of content and analyzing it from various dimensions

2. Appropriate way for revising and consolidating the content

3. Makes us understand the relevance of the things learned

4. Very interactive and group discussion helps in team building

5. Long term retention of knowledge which would help in practical application in clinics

\section{Limitations of clinical reasoning sessions (only two responses were obtained after collation and arranged according to frequency)}

1. Doesn't provide room for adequate theoretical knowledge

2. Can be taken to the wards and exposed to real patients

\section{Discussion}

Enabling students to transfer their anatomical knowledge from the classroom to the clinical settings is one of the biggest challenges for contemporary anatomists. One of the core entrustable professional activities expected out of a graduate medical student is to prioritize a differential diagnosis following a clinical encounter [24]. The key aim for designing the clinico-anatomical case vignette session is to structure the clinical reasoning skills right from the pre-clinical phase of medical education. The process of clinical reasoning, which is indeed complex, is a balance between pattern recognition and hypothetico-deductive approaches. Even though novice learners use hypothetico-deductive approach to figure out the diagnosis in the context of undifferentiated illness, the ability to filter the differentials and reason out the provisional diagnosis varies according to the individual attributes of clinical reasoning [25]. In our sessions, we adopted 'think-pair-share' approach so that the common clinical reasoning difficulties such as difficulty in data gathering, synthesis, premature closure, synthesis and prioritizing are avoided to possible extent. Similarly, the cases were presented using 'serial-cue' approach whereby the chief complaint is initially provided and hypothesis generated is subsequently tested based on the further information. An experimental study by Mylopoulos and Woods [26]. showed that integration of clinical manifestations alongside with basic science mechanisms might support 'preparation for future learning' and this is imperative for graduate medical students to learn from experiences in future. Similarly, the present study also provides evidence for improvement in the ability to solve post-test questions among intervention group students when compared to control group.

During the course of vignette, we exposed students to a range of clinical possibilities including complaints and abnormal clinical presentations. When students pair chief complaints with currently acquired anatomical knowledge, the quality of integration would be better in terms of knowledge networks $[27,28]$. When students practice the clinical scenarios in such virtual yet non-graded environment, students gain familiarity with clinical care environment [29]. In the present study, majority of the students believed that the case vignettes are relevant to the context and spanned across various disciplines. It is important to ascertain the complexity level of the case scenarios because if students had not possessed the adequate level of knowledge structures, the clinical reasoning sessions would not culminate in causal structural models and thereby not improving the diagnostic abilities.

We had obtained equivocal response regarding the prompts used amidst the clinical vignettes. Prompts are specific instructions that helps the students to navigate the content in a specific guided way [30]. Out of the various types of prompts, we used 'gap-filling' prompts which would support the cognitive process of students in generating the principal justification to the underlying anatomical concepts of case vignette [31]. Nevertheless, we could observe that a proportion of students were swift in utilizing the prompts compared to others, which could be due to their richer causalstructural knowledge models. This could have impeded the diagnostic process of others in 'think-pair-share' groups and resulted in equivocal responses.

Majority of the students admitted that the clinical reasoning sessions have increased their diagnostic and lateral thinking abilities. This could be compared with previous documentation by Mamede et al. [32] which showed that 
structural reflection on clinical cases was beneficial in fostering clinical diagnosis compared to the usual pattern of generating readymade differential diagnosis. When students perform structured clinical reasoning, they try to compare and contrast various alternate possibilities and generate nascent illness scripts. Thus, the process would enable significant 'transfer of learning' while solving similar cases in the clinical environment [33]. Jain et al. [34] conducted an open labelled randomized control experiment to test the effectiveness of a six step pneumonic centred clinical reasoning model (SNAPPS) in improving the clinical reasoning ability of postgraduates. They found that students in SNAPPS group were clearer in generating diagnostic hypothesis and because of this, they could compare and contrast the differential diagnosis in a better way compared to control group. On the other hand, the control group struggled in generating the diagnostic hypothesis and prematurely closed the diagnosis moving on to management issues. Therefore, the ability to compare and contrast the varied clinical presentations is one of the valuable clinical reasoning aids in the preclinical phase of medical education.

Despite of the wider positive response towards clinical reasoning sessions, our concern is regarding $30 \%$ students who hadn't benefited significantly (Fig. 5). Based upon the observatory data of the investigators, we could make out three reasons. Firstly, the development of schemas depends upon the curiosity and motivation demonstrated by the students which hugely varies. Secondly, the processing of incoming information based upon modelled reflection depends upon the level of pre-existing knowledge and students with limited ability to match their knowledge, would find the exercise as tiring process. Thirdly, some of these students would have experienced extraneous cognitive load which might have impeded the learning. We second the comment by Schmidt and Boshuizen [35] who stated that verbalization and interaction of thoughts is the prime requirement for gaining clinical reasoning skill and it is these thoughts which later become encapsulated and tacit schema with practice. Indeed, the outcome of the clinical reasoning sessions depends upon the tripartite role of student, facilitator and clinical case content as such.

\section{Limitations}

The pilot trial involved first year medical students belonging to single institution and the results cannot be generalized to all educational settings. Secondly, considering the fact that all participants are novice learners, we hadn't adopted any grading scheme for defining the development of clinical reasoning and the perceptions obtained has elements of subjectivity. Thirdly, we couldn't follow the standard assessment typologies for clinical reasoning acquisition because the number of permissible sessions were limited and the process is an evolving one. The reasoning skills, which we intend to develop, is an additional entity delinked from the summative examinations and therefore, the immediate benefit wasn't sensed by few students. We felt that incorporating it as a part of curriculum would still more yield solid benefits. Fourthly, due to practical restrictions, we could not adopt 'think-aloud' protocol which is by far the effective way for enhancing clinical reasoning skills. Because of this, we could not exactly capture the quality of interaction taking place. Finally, students have attended few problem based learning sessions in physiology concurrently. Thus, the diagnostic thinking and development of clinical reasoning could not be completely attributed as outcomes of our pedagogy.

In conclusion, despite the limitations stated above, conducting exclusive clinical reasoning sessions using clinicoanatomical case vignettes helps in providing explicit steps for the students in approaching illness scripts, handling uncertainties, developing probabilistic hypotheses and developing necessary probes. The novice learners could therefore elaborate causal-structural networks for comprehending the anatomical concepts behind diseases and analyse the symptoms in different view. This also highlights the need for developing reasoning as a competency in graduate medical curriculum which enables the usage of biomedical knowledge in clinical practice. At the same time, standardized assessment tools need to be developed for measuring the threshold level of clinical reasoning skills. In our future studies, we would like to explore newer temporal models for conceptualizing clinical reasoning and means for developing it right from first year to the final year of medical education in limited resource settings.

\section{ORCID}

Dinesh Kumar. V: https://orcid.org/0000-0001-8234-2829

Rajprasath R: https://orcid.org/0000-0003-0902-364X

N.A. Priyadharshini:

https://orcid.org/0000-0002-4232-9029

Magi Murugan: https://orcid.org/0000-0002-8458-479X

Rema Devi: https://orcid.org/0000-0003-4840-0313 


\section{Author Contributions}

Conceptualization: DKV, RR, NAP, MM. Data acquisition: RR, NAP, MM, RD. Data analysis or interpretation: DKV, RR, NAP, MM. Drafting of the manuscript: DKV, RR, MM. Critical revision of the manuscript: DKV, RR, MM, RD. Approval of the final version of the manuscript: all authors.

\section{Conflicts of Interest}

No potential conflict of interest relevant to this article was reported.

\section{References}

1. Norman G. Research in clinical reasoning: past history and current trends. Med Educ 2005;39:418-27.

2. Kassirer JP. Teaching clinical reasoning: case-based and coached. Acad Med 2010;85:1118-24.

3. van Gog T, Ericsson KA, Rikers RMJP, Paas F. Instructional design for advanced learners: establishing connections between the theoretical frameworks of cognitive load and deliberate practice. Educ Technol Res Dev 2005;53:73-81.

4. van Merriënboer JJG, Sweller J. Cognitive load theory and complex learning: recent developments and future directions. Educ Psychol Rev 2005;17:147-77.

5. Allchin D. Problem- and case-based learning in science: an introduction to distinctions, values, and outcomes. CBE Life Sci Educ 2013;12:364-72.

6. Dammers J, Spencer J, Thomas M. Using real patients in problem-based learning: students' comments on the value of using real, as opposed to paper cases, in a problem-based learning module in general practice. Med Educ 2001;35:27-34.

7. Waechter D. Liaison Committee on Medical Education glossary of terms for LCME accreditation standards and elements 2015-2016. Washington, DC: LCME; 2015. 10 p.

8. Dalton L, Gee T, Levett-Jones T. Using clinical reasoning and simulation-based education to 'flip' the Enrolled Nurse curriculum. Aust J Adv Nurs 2015;33:29-35.

9. Kong LN, Qin B, Zhou YQ, Mou SY, Gao HM. The effectiveness of problem-based learning on development of nursing students' critical thinking: a systematic review and meta-analysis. Int J Nurs Stud 2014;51:458-69.

10. Bowen JL. Educational strategies to promote clinical diagnostic reasoning. N Engl J Med 2006;355:2217-25.

11. Hall KH. Reviewing intuitive decision-making and uncertainty: the implications for medical education. Med Educ 2002;36: 216-24.

12. Dawson NV. Physician judgment in clinical settings: methodological influences and cognitive performance. Clin Chem
1993;39:1468-78; discussion 1478-80.

13. Fischhoff B. Hindsight not equal to foresight: the effect of outcome knowledge on judgment under uncertainty. Qual Saf Health Care. 2003;12:304-11; discussion 311-2.

14. Dawson NV, Arkes HR, Siciliano C, Blinkhorn R, Lakshmanan M, Petrelli M. Hindsight bias: an impediment to accurate probability estimation in clinicopathologic conferences. Med Decis Making 1988;8:259-64.

15. Hassan S. About clinicopathological conference and its' practice in the School of Medical Sciences, USM. Malays J Med Sci 2006;13:7-10.

16. Christensen N, Black L, Furze J, Huhn K, Vendrely A, Wainwright S. Clinical reasoning: survey of teaching methods, integration, and assessment in entry-level physical therapist academic education. Phys Ther 2017;97:175-86.

17. Boshuizen HPA, Schmidt HG. On the role of biomedical knowledge in clinical reasoning by experts, intermediates and novices. Cogn Sci 1992;16:153-84.

18. Linsen A, Elshout G, Pols D, Zwaan L, Mamede S. Education in clinical reasoning: an experimental study on strategies to foster novice medical students' engagement in learning activities. Health Prof Educ 2018;4:86-96.

19. Mamede S, Figueiredo-Soares T, Elói Santos SM, de Faria RMD, Schmidt HG, van Gog T. Fostering novice students' diagnostic ability: the value of guiding deliberate reflection. Med Educ 2019;53:628-37.

20. Mamede S, Schmidt HG, Penaforte JC. Effects of reflective practice on the accuracy of medical diagnoses. Med Educ 2008; 42:468-75

21. Kreiter CD, Bergus G. The validity of performance-based measures of clinical reasoning and alternative approaches. Med Educ 2009;43:320-5.

22. Kumar D, Rajprasath R, Murugan M. Integrating clinical reasoning principles in case-based learning sessions for first-year medical students: lessons learned. Res Dev Med Educ 2019;8: 20-3.

23. Elstein AS, Shulman LS, Sprafka SA. Medical problem solving: an analysis of clinical reasoning. Cambridge, MA: Harvard University Press; 1978.

24. Ten Cate O. Trusting graduates to enter residency: what does it take? J Grad Med Educ 2014;6:7-10.

25. Wolpaw T, Papp KK, Bordage G. Using SNAPPS to facilitate the expression of clinical reasoning and uncertainties: a randomized comparison group trial. Acad Med 2009;84:517-24.

26. Mylopoulos M, Woods N. Preparing medical students for future learning using basic science instruction. Med Educ 2014; 48:667-73

27. van Gessel E, Nendaz MR, Vermeulen B, Junod A, Vu NV. Development of clinical reasoning from the basic sciences to the clerkships: a longitudinal assessment of medical students' needs and self-perception after a transitional learning unit. Med Educ 2003;37:966-74.

28. Barfield LC, Taylor BR, Santen SA. Learning physical diagnosis: a new paradigm. Med Educ 2007;41:1097. 
29. Atherley AE, Hambleton IR, Unwin N, George C, Lashley PM, Taylor CG Jr. Exploring the transition of undergraduate medical students into a clinical clerkship using organizational socialization theory. Perspect Med Educ 2016;5:78-87.

30. Schworm S, Renkl A. Learning argumentation skills through the use of prompts for self-explaining examples. J Educ Psychol 2007;99:285-96.

31. Nokes TJ, Hausmann RGM, Vanlehn K, Gershman S. Testing the instructional fit hypothesis: the case of self-explanation prompts. Instr Sci 2011;39:645-66.

32. Mamede S, van Gog T, Sampaio AM, de Faria RM, Maria JP, Schmidt HG. How can students' diagnostic competence benefit most from practice with clinical cases? The effects of structured reflection on future diagnosis of the same and novel diseases. Acad Med 2014;89:121-7.

33. Nishigori H, Masuda K, Kikukawa M, Kawashima A, Yudkowsky R, Bordage G, Otaki J. A model teaching session for the hypothesis-driven physical examination. Med Teach 2011;33: 410-7.

34. Jain V, Rao S, Jinadani M. Effectiveness of SNAPPS for improving clinical reasoning in postgraduates: randomized controlled trial. BMC Med Educ 2019;19:224.

35. Schmidt HG, Boshuizen HPA. On acquiring expertise in medicine. Educ Psychol Rev 1993;5:205-21. 